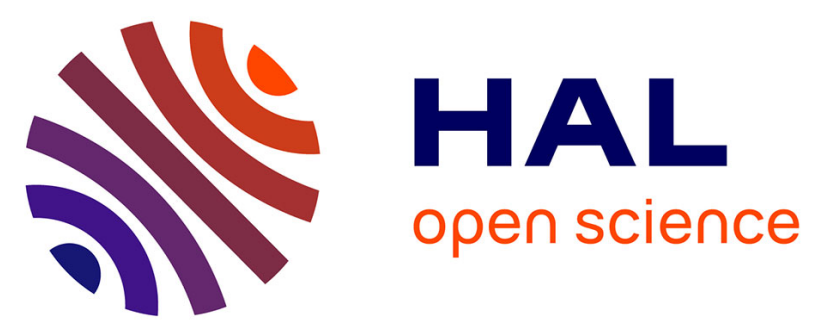

\title{
Voices' inter-animation detection with ReaderBench. Modelling and assessing polyphony in CSCL chats as voice synergy
}

\author{
Mihai Dascalu, Stefan Trausan-Matu, Philippe Dessus
}

\section{To cite this version:}

Mihai Dascalu, Stefan Trausan-Matu, Philippe Dessus. Voices' inter-animation detection with ReaderBench. Modelling and assessing polyphony in CSCL chats as voice synergy. Proc. 2nd Int. Workshop on Semantic and Collaborative Technologies for the Web, in conjunction with 2nd Int. Conf. on Systems and Computer Science (ICSCS'2013), Aug 2013, France. pp.280-285. hal-00906939

\section{HAL Id: hal-00906939 \\ https://hal.science/hal-00906939}

Submitted on 20 Nov 2013

HAL is a multi-disciplinary open access archive for the deposit and dissemination of scientific research documents, whether they are published or not. The documents may come from teaching and research institutions in France or abroad, or from public or private research centers.
L'archive ouverte pluridisciplinaire HAL, est destinée au dépôt et à la diffusion de documents scientifiques de niveau recherche, publiés ou non, émanant des établissements d'enseignement et de recherche français ou étrangers, des laboratoires publics ou privés. 


\title{
Voices' Inter-Animation Detection with ReaderBench Modelling and Assessing Polyphony in CSCL Chats as Voice Synergy
}

\author{
Mihai Dascalu, Stefan Trausan-Matu and Philippe Dessus
}

\begin{abstract}
Starting from dialogism in which every act is perceived as a dialogue, we shift the perspective towards multiparticipant chat conversations from Computer Supported Collaborative Learning in which ideas, points of view or more generally put voices interact, inter-animate and generate the context of a conversation. Within this perspective of discourse analysis, we introduce an implemented framework, ReaderBench, for modeling and automatically evaluating polyphony that emerges as an overlap or synergy of voices. Moreover, multiple evaluation factors were analyzed for quantifying the importance of a voice and various functions were experimented to best reflect the synergic effect of cooccurring voices for modeling the underlying discourse structure.
\end{abstract}

\section{BAKHTIN'S DIALOGISM AS A FRAMEWORK FOR CSCL}

Dialogism was introduced by the Russian philosopher Mikhail Bakhtin [1,2] and covers a broader, more abstract and comprehensive sense of dialogue that is reflected in "any kind of human sense-making, semiotic practice, action, interaction, thinking or communication, as long as these phenomena are 'dialogically' or 'dialogistically' understood" [3]. This provides a differentiation criteria in terms of the classic dialogue theories that are focused on the interactions between two or more individuals, mutually present in realtime or with accepted delayed responses, using different communication channels (of particular interest here are the computer-supported "dialogues").

With regards to Computer Supported Collaborative Learning (CSCL), dialogism was proposed by Koschmann [4] as a paradigm for CSCL, its key features being multivocality and polyphony. Wegerif [5] also considered dialogism as a theoretical starting point that can be used for developing tools to teach thinking skills. Moreover, Wegerif believes that inter-animation is a key component for the success of collaborative learning. Following these ideas, Trausan-Matu introduced the polyphonic theory, model and analysis method [6-9] for CSCL.

M. Dascalu is with the Department of Computer Science, University "Politehnica" of Bucharest, 313 Splaiul Independentei, Bucharest, Romania (phone: +40 726111 091; e-mail: mihai.dascalu@cs.pub.ro).

S. Trausan-Matu is with the Department of Computer Science, University "Politehnica" of Bucharest, 313 Splaiul Independentei, Bucharest, Romania (e-mail: stefan.trausan@cs.pub.ro).

P. Dessus is with the Laboratory of Educational Sciences, University Grenoble Alpes, 1251 av. Centrale, Grenoble CEDEX 9, France (e-mail: philippe.dessus@upmf-grenoble.fr).
In order to properly introduce the polyphonic model presented in detail in the following section and later on used within our implemented system, ReaderBench [10,11], we should first introduce the three core and inter-dependent concepts of discourse analysis: utterances briefly defined as units of analysis, voices as distinctive points of view emerging from the ongoing discussion and echoes as the replication of a certain voice with further implications in the discourse. Similar to some extent to the dialogical discourse analysis proposed by Linell [12] and Marková, et al. [13] focused on the dynamics and recurrence of topics ('themes') and their rhetoric expressions (e.g., analogies, distinctions, metaphors, use of quotes) [13], all computational perspectives are inevitably limiting while analyzing the dialogical nature of discourse: "it is indeed impossible to be 'completely dialogical', if one wants to be systematic and contribute to a cumulative scientific endeavor" [3]. This also augments the duality between individual involvement and actual collaboration throughout a given CSCL conversation, as it is impossible to focus on both the animation of other participants and sustainably providing meaningful utterances; in the end, a balance needs to be achieved between individuals, without encouraging domination of the discourse in terms of participation.

The paper continues with details of the underlying polyphonic model of discourse analysis, leading to the introduction of ReaderBench. The third section is centered on the analysis of textual cohesion, considered central within discourse analysis. Then we shift the point of interest towards reading strategies and assessing textual complexity. Each of the three latter sections is accompanied by a validation with ReaderBench.

\section{THE POLYPHONIC MODEL OF DISCOURSE ANALYSIS}

The polyphonic theory $[6-8,14,15]$ follows the ideas of Koschmann [4] and Wegerif [16] and investigates how Bakhtin's theory of polyphony and inter-animation $[1,2]$ can be used for analyzing the discourse in chat conversations with multiple participants. In phone and face-to-face dialogs only one person usually speaks at a given moment in time, generating a single thread of discussion. This is, of course, determined by the physical, acoustical constraints (if two or more persons are speaking in the same moment, it is impossible to understand something). In chat environments, 
such the one used in the Virtual Math Teams (VMT) project [17], any number of participants may write utterances at the same time because it offers explicit referencing facilities that allow the users to indicate to what previous utterance(s) they refer to. This facility is extremely important in chat conversations with more than two participants because it allows the existence of several discussion threads in parallel. Moreover, the co-occurrence of several threads gives birth to inter-animation, a phenomenon similar to polyphony, where several voices jointly play a coherent piece as a whole $[8,9]$.

Bakhtin [2] emphasized that polyphony occurs in any text. He considered that dialog characterizes any text, that "our speech, that is, all our utterances (including creative works), is filled with others' words" [18]. The voice becomes a central concept, has a more complex meaning. A voice is not limited to the acoustic dimension, it may be considered as a particular position, which may be taken by one or more persons when emitting an utterance $[8,9]$, which may have both explicit, similar to those provided by the VMT chat environment [17], and implicit links (for example, lexical chains, co-references or argumentation links) and influence other voices. Each utterance is filled with 'overtones' of other utterances [17]. Moreover, by the simple fact that they co-occur, voices are permanently inter-animating [9], entering in competition, generating multi-vocality in any conversation and even in any text (in Bakhtin's dialogic theory everything is a dialog) or, as Bakhtin calls it, a "heteroglossia, which grows as long as language is alive" [1].

The ideas of Bakhtin are based on a musical metaphor for discourse and for learning: "the voices of others become woven into what we say, write, and think" [4]. Therefore, for analyzing discourse in chats the aim shifts towards investigating how voices are woven, how themes and voices inter-animate in a polyphonic way [9]. This is important not only for understanding how meaning is created but also for trying to design tools for support and evaluation. Fig 1 presents the inter-animation of voices within a chat conversation and their evolution in time, following a pattern first described by Trausan-Matu, et al. [6]; the longest two voices are represented by the linked curly lines. As it can be observed, several threads can co-appear in parallel and even the same participant may participate to more than one discussion thread within a given timeframe (e.g. John, at utterance 19, approves and elaborates Tim's intervention, while the following utterance represents an approval of Adrian's utterance 18) [8]. Therefore, this co-presence of multiple discussion threads and their inter-influences models voice inter-animation towards achieving polyphony.

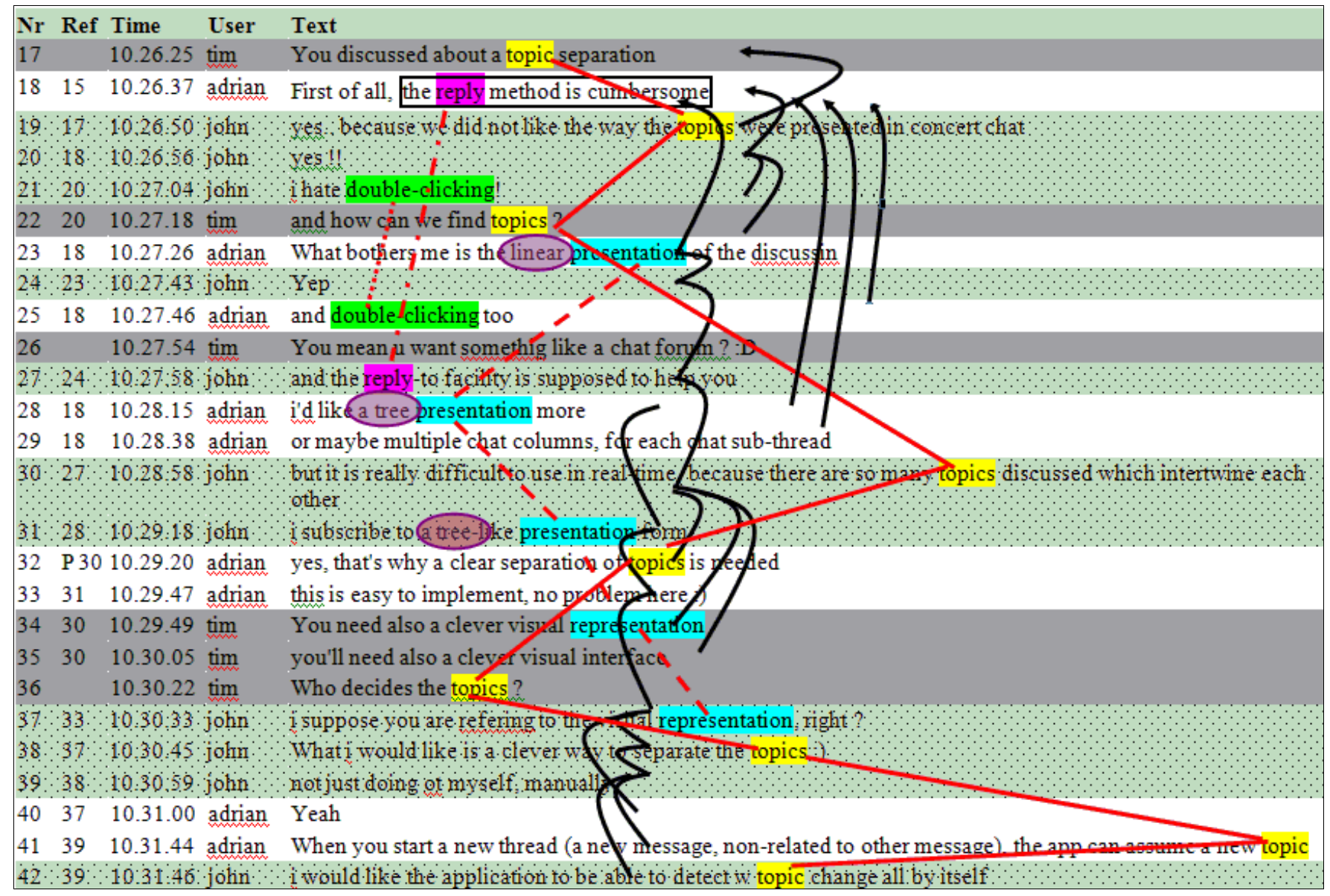

Figure 1. Inter-animation of voices within a chat [9]. 
The polyphonic model focuses on the idea of identifying voices in the analysis of discourse and building an internal graph-based representation, whether we are focusing on the utterance graph [19] or the cohesion graph [10, 20]. For this aim, links between utterances are analyzed using adjacency pairs, repetitions, lexical chains, speech and argumentation acts or cohesive links, a graph is built from which discussion threads are identified. Nevertheless, in both internal representations, lexical or semantic cohesion between any two utterances seen as explicit communicative acts can be considered the central liaison between the analysis elements within the graph. Cohesion can be expressed as the "distance" between the utterance boundaries [21] and can be computed by various means of semantic similarity, including semantic distances in ontologies [22], latent vector space representations [23] or topic models [24].

As the initial polyphonic model used the utterance graph [19] and the cohesion graph [10, 20], we will focus on providing a comprehensive view of the polyphonic model, using as underlying representation the utterance graph. This internal structure is built upon two types of links between utterances: explicit and implicit. Participants manually add explicit links during their chat sessions by using a facility from the conversation environment - e.g., ConcertChat [25]. On the other hand, implicit links are automatically identified by means of co-references, repetitions, lexical chains and inter-animation patterns $[6,26]$. In the resulted graph, each utterance is a node and the weights of edges are given by the similarity between the utterances. The orientation of each edge follows the timeline of the chat and the evolution of the discussion in time. Starting from the previous graph, a thread can be easily identified as a logical succession of explicitly or implicitly inter-linked utterances. Moreover, the primary extension of each utterance is its inner voice that inter-twines with other voices from the same thread or from different ones, but with less strength. A new intervention or a new utterance in terms of units of analysis can be clearly expressed as a voice and aspects that need to be addressed include: degree of interconnection in terms of cohesion with other utterances, relevance within the discourse or future impact in the overall discussion.

Starting from Bakhtin [2] perspective of discourse analysis, each identified voice may become more or less powerful than the others and may influence the others. Among chat voices there are sequential and transversal relations, highlighting a specific point of view in a counterpointal way, as mentioned in previous work [9, 14]. The co-occurrence of several voices which enter in dialogue is a phenomenon considered by Bakhtin to be universal, present in any text, not only in conversations: "Life by its very nature is dialogic ... when dialogue ends, everything ends" [2]. Bakhtin moves the focus of analysis from sentences to utterances in an extended way, in which even an essay contains utterances and is, at its turn, an utterance.
Moreover, each utterance is filled with 'overtones' that contain the echoes and influence of other previous utterances.

A voice is generated by an utterance with effects (echoes) on the subsequent utterances via explicit and implicit links. Moreover, by the simple fact that they co-occur, voices are permanently interacting, overlapping and inter-animating, entering in competition, and generating multivocality in any conversation. The ideal situation of a successful conversation or a coherent discourse is achieved when the voices are entering inter-animation patterns based on the discussion threads they are part of [6].

\section{COMPUTATIONAL DIALOGISM AND VOICE INTER-ANIMATION WITHIN READERBENCH}

The key element in terms of voice identification resides in building lexical chains and merging them into semantic chains through cohesion. Due to the limitation of discovering lexical chains [27] through semantic distances in WordNet [28] or WOLF [29] that only consider words having the same part-of-speech, the merge step is essential as it enables consideration of different parts-of-speech and unites groups of concepts based on identical lemmas or high cohesion values. In this context, we have proposed an iterative algorithm similar to an agglomerative hierarchical clustering algorithm [30] that starts with the identified lexical chains seen as groups of already clustered words and uses as distance function the cohesion between the corresponding groups of words, if this value is greater than an imposed threshold, in order to merge clusters.

As semantic chains span across the discourse, the context generated by the co-occurrence or repetitions of tightly cohesive concepts is similar to the longitudinal dimension of voices. Echoes can be highlighted through cohesion based on semantic relationship and attenuation is reflected in the considered distance between utterances. Moreover, by intertwining different semantic chains within the same textual fragment (sentence, utterance or paragraph) we are able to better grasp the transversal dimension of voice interanimation. Therefore, after manually selecting the voices of interest, the user can visualize the conversation as an overlap of co-occurring semantic chains that induce polyphony (see Fig. 2). A voice is displayed within the interface as the 3 most dominant concepts (word lemmas) and its occurrences throughout the conversation are marked accordingly to the overall timeframe. Different speakers that uttered a particular voice are demarcated with randomly assigned colors, consistent throughout a conversation for each participant. Each utterance may incorporate more than a single voice, as it may include, in addition to the current participant's voice, at least one other, an alien voice $[1,7]$, identified through semantic chains and cohesive links. 


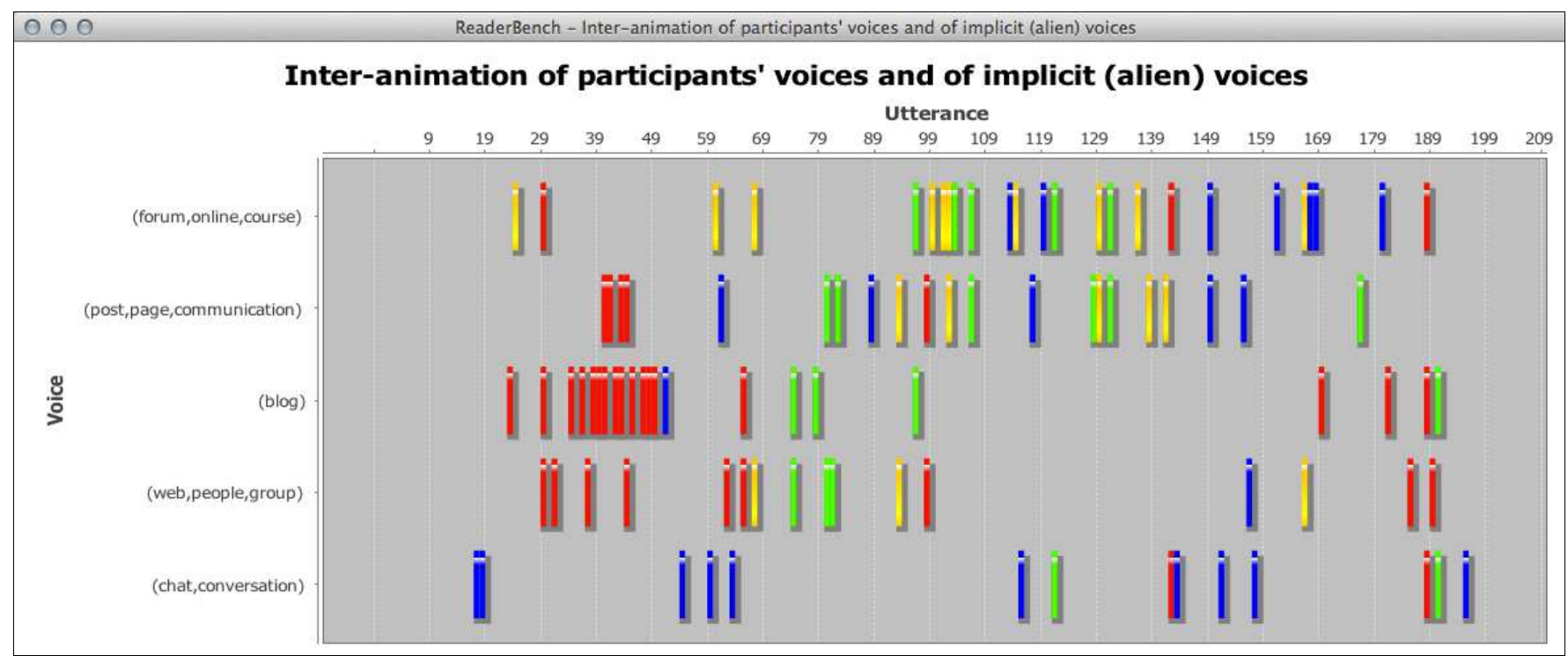

Figure 2. Chat voice inter-animation visualization in ReaderBench covering participants' voices and implicit (alien) voices. The chart follows the conversation timeline expressed in utterance identifiers and depicts the occurrences of the 5 most dominant voices: $1 /$ (forum, online, course), $2 /$ (post, page, communication), 3/ (blog), 4/ (web, people, group) and 5/ (chat, conversation). Each of the 4 chat participants has a corresponding color and each voice occurrence reflects the speaker's assigned color.

In order to better grasp the importance of each voice within the discourse, we have devised a series of indicators, some inspired from 'rhythmanalysis' [31] and 'polyrhythm' [32]: $1 /$ the number of contained words as a pure quantitative factor, $2 /$ the cumulative scores of the analysis elements that provides a broader image of the importance of the context of their occurrence (qualitative oriented) and 3/ the recurrence of voices seen as the distance between two analysis elements in which consecutive occurrences of the voice appear, inspired from rhythm analysis.

Moreover, in accordance to Miller's law [33], we have applied a simple moving average [34] on the voice distribution for five datum points representing consecutive utterances (or sentences in the case of general texts), with a split horizon of one minute between adjacent interventions (only for chat-based conversations where the timestamp of each utterance is used). In other words, we weight the importance of each concept occurrence over 5 adjacent utterances, if no break in the discourse is larger than an imposed, experimentally determined threshold of one minute. Exceeding this value would clearly mark a stopping point in the overall chat conversation, making unnecessary the expansion of the singular occurrence of the voice over this break. This step of smoothing the initial discrete voice distribution plays a central role in subsequent processing as the expanded context of a voice's occurrence is much more significant than the sole consideration of the concept uttered by a participant in a given intervention. In this particular case, entropy [35] has been applied on the smoothed distribution in order to highlight discrepancies of voice occurrences throughout the entire conversation.

By considering all the previous factors used to estimate the importance of a voice, Table 1 presents the crosscorrelations when considering a conversation of approximately 400 interventions and all 57 automatically identified voices, with the sole constraint that each voice had to include at least 3 word occurrences in order to have a quantifiable overall impact. Overall, all factors, besides recurrence, correlate positively and can be used to estimate the overall impact of a voice within the conversation, whereas recurrence is more specific and can be used to pinpoint whether the concepts pertaining to a voice are collocated or are more equally dispersed throughout the discourse. Nevertheless, small correlation values are acceptable as our aim was to identify meaningful factors that can be used to better characterize a voice's importance. Further evaluations need to be performed in order to determine the most representative factors, but our aim was to identify specific measures of evaluation that are generated as effects of different underlying assessment factors (e.g., the use of the number of utterances in which the voices occurred or of statistics applied on the initial distribution would have been inappropriate as all these factors would have been directly linked to the number of words within the semantic chain).

TABLE I. CROSS-CORRELATION MATRIX FOR VOICE ANALYSIS FACTORS.

\begin{tabular}{|l|l|l|l|l|l|}
\hline \multicolumn{1}{|c|}{ Text } & $\mathbf{1}$ & $\mathbf{2}$ & $\mathbf{3}$ & $\mathbf{4}$ & $\mathbf{5}$ \\
\hline $\begin{array}{l}\text { 1. Number of words within } \\
\text { the semantic chain }\end{array}$ & 1 & & & & \\
\hline $\begin{array}{l}\text { 2. Average utterance } \\
\text { importance scores }\end{array}$ & .20 & 1 & & & \\
\hline $\begin{array}{l}\text { 3. Entropy applied on the } \\
\text { utterance moving average }\end{array}$ & .77 & .26 & 1 & & \\
\hline 4. Recurrence Average & -.44 & -.20 & -.68 & 1 & \\
\hline $\begin{array}{l}\text { 5. Recurrence standard } \\
\text { deviation }\end{array}$ & -.35 & -.08 & -.46 & 0.67 & 1 \\
\hline
\end{tabular}


As voice synergy emerges as a measure of co-occurrence of semantic chains, mutual information [36] can be used to quantify the global effect of voice overlapping between any pairs of voices. Moreover, by applying pointwise mutual information (PMI) [37] between the moving averages of all pairs of voice distributions that appear in a given context of five analysis elements, we obtain a local degree of voice inter-weaving or overlap. In order to better grasp the underlying reason of using PMI, we have presented in Fig. 3 three progressive measures for synergy.

The first and the simplest, the actual number of voices (co-)occurring, is misleading as we encounter a lot of singular values (meaningless as only one voice is present) and double ones, which are also not that interesting in observing the global trend. Also, the first spike with a value of 3 is locally representative, but since it's isolated from the rest of the conversation, its importance should be mediated globally. The second, the cumulated moving average, is better as the smoothing effect has a positive impact on the overall evolution. Nevertheless, it is misleading in some cases - e.g., the maximum value is obtained around utterance 40 where the conversation is dominated by one participant and one voice, but by being so strong, even the smoothed effect is artificially augmented.

The third, the average PMI applied on the moving averages, grasps best the synergic zones: e.g., just after utterance 60 we have all five selected voices co-occurring, between 95 and 100 an overlap of four voices, the first two being well represented and dominant, and just before utterance 190 we also have four co-occurring voices. Therefore, by observing the evolution of PMI using a sliding window that follows the conversation flow, we obtain a trend in terms of synergy that can be later on generalized to Bakhtin's polyphony [2].

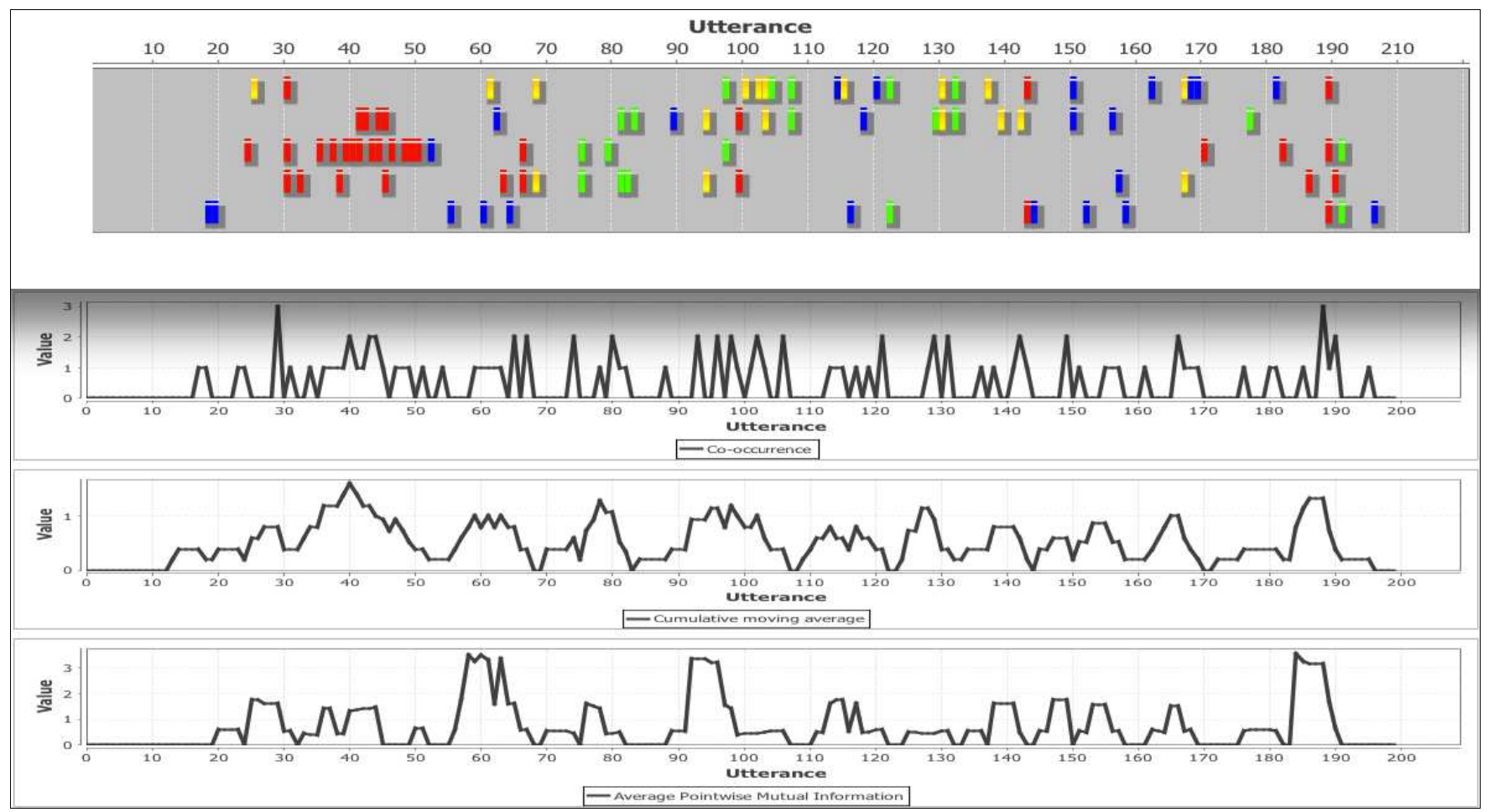

Figure 3. Evolution of voice synergy throughout the conversation. a. Voice visualization as time evolution (baseline for comparison); b. Number of occurrences; c. Evolution of cumulated moving average; d. Average pointwise mutual information.

\section{CONCLUSIONS}

By considering dialogism as a framework for CSCL and by modeling the discourse structure through polyphony, we were able to capture within ReaderBench the thematic structure of a conversation perceived as the synergy of voices, extracted as semantic chains. Nevertheless, within our implemented system we opted for graphically presenting the evolution of voice synergy instead of polyphony because our computational model uses co-occurrences and overlaps of voices within a given context. In order to emphasize the effect of inter-animation that would induce true polyphony, we envisage the use of argumentation acts and patterns [38] for highlighting the interdependencies between voices and how a particular voice can shed light on another. Later on, by integrating mood assessment specific metrics [39] we strive to differentiate centrifugal and centripetal forces.

\section{ACKNOWLEDGMENTS}

This research was supported by an Agence Nationale de la Recherche (DEVCOMP) grant, by the 264207 ERRICEmpowering Romanian Research on Intelligent Information Technologies/FP7-REGPOT-2010-1 and the POSDRU/107/ 1.5/S/76909 Harnessing human capital in research through doctoral scholarships (ValueDoc) projects. 


\section{REFERENCES}

[1] M. M. Bakhtin, The dialogic imagination: Four essays. Austin and London: The University of Texas Press, 1981.

[2] M. M. Bakhtin, Problems of Dostoevsky's poetics. Minneapolis: University of Minnesota Press, 1984.

[3] P. Linell, Rethinking language, mind, and world dialogically: Interactional and contextual theories of human sense-making. Information Age Publishing: Charlotte, NC, 2009.

[4] T. Koschmann, "Toward a dialogic theory of learning: Bakhtin's contribution to understanding learning in settings of collaboration," in Int. Conf. on Computer Support for Collaborative Learning (CSCL'99), Palo Alto, 1999, pp. 308-313.

[5] R. Wegerif, "A Dialogical Understanding of the Relationship between CSCL and Teaching Thinking Skills," International Journal of Computer-Supported Collaborative Learning, vol. 1, pp. 143-157, 2006.

[6] S. Trausan-Matu, G. Stahl, and A. Zemel, "Polyphonic Interanimation in Collaborative Problem Solving Chats," Drexel University, Philadelphia2005.

[7] S. Trausan-Matu and G. Stahl, "Polyphonic inter-animation of voices in chats," in CSCL'07 Workshop on Chat Analysis in Virtual Math Teams, New Brunwick, NJ, 2007.

[8] S. Trausan-Matu, "The Polyphonic Model of Hybrid and Collaborative Learning," in Handbook of Research on Hybrid Learning Models: Advanced Tools, Technologies, and Applications, F. Wang, L., J. Fong., and R. C. Kwan, Eds., ed Hershey, NY: Information Science Publishing, 2010, pp. 466-486.

[9] S. Trausan-Matu, G. Stahl, and J. Sarmiento, "Supporting polyphonic collaborative learning," Indiana University Press, E-service Journal, vol. 6, pp. 58-74, 2007.

[10] M. Dascalu, P. Dessus, S. Trausan-Matu, M. Bianco, and A. Nardy, "ReaderBench, an Environment for Analyzing Text Complexity and Reading Strategies," in 16th Int. Conf. on Artificial Intelligence in Education (AIED 2013), Memphis, USA, 2013, pp. 379-388.

[11] M. Dascalu, S. Trausan-Matu, and P. Dessus, "Cohesion-based Analysis of CSCL Conversations: Holistic and Individual Perspectives," in 10th Int. Conf. on Computer-Supported Collaborative Learning (CSCL 2013), Madison, USA, 2013, pp. 145152.

[12] P. Linell, "A dialogical conception of focus groups and social representations," in Socio-cultural theory and methods: An anthology, U. S. Larsoon, Ed., ed Trollhättan, Sweeden: University of Trollhättan/Uddevalla, 2001.

[13] I. Marková, P. Linell, M. Grossen, and A. Salazar Orvig, Dialogue in focus groups: Exploring socially shared knowledge. London, UK: Equinox, 2007.

[14] S. Trausan-Matu and T. Rebedea, "Polyphonic Inter-Animation of Voices in VMT," in Studying Virtual Math Teams, G. Stahl, Ed., ed Boston, MA: Springer, 2009, pp. 451-473.

[15] S. Trausan-Matu, T. Rebedea, and M. Dascalu, "Analysis of discourse in collaborative Learning Chat Conversations with Multiple Participants," in Multilinguality and Interoperability in Language Processing with Emphasis on Romanian, D. Tufis and C. Forascu, Eds., ed Bucharest, Romania: Editura Academiei, 2010, pp. 313-330.

[16] R. Wegerif, "A dialogical understanding of the relationship between CSCL and teaching thinking skills," in Conf. on Computer Supported Collaborative Learning 2005: The Next 10 Years! (CSCL'05), Taipei, Taiwan, 2005.

[17] G. Stahl, Studying Virtual Math Teams. New York, NY: Springer, 2009.

[18] M. M. Bakhtin, Speech genres and other late essays. Austin: University of Texas, 1986.

[19] S. Trausan-Matu, T. Rebedea, A. Dragan, and C. Alexandru, "Visualisation of learners' contributions in chat conversations," in
Blended learning, J. Fong and F. L. Wang, Eds., ed Singapour: Pearson/Prentice Hall, 2007, pp. 217-226.

[20] S. Trausan-Matu, M. Dascalu, and P. Dessus, "Textual Complexity and Discourse Structure in Computer-Supported Collaborative Learning," in 11th Int. Conf. on Intelligent Tutoring Systems (ITS 2012), Chania, Grece, 2012, pp. 352-357.

[21] A. Dong, "The latent semantic approach to studying design team communication," Design Studies, vol. 26, pp. 445-461, 2005.

[22] A. Budanitsky and G. Hirst, "Evaluating WordNet-based Measures of Lexical Semantic Relatedness," Computational Linguistics, vol. 32, pp. 13-47, 2006.

[23] T. K. Landauer and S. T. Dumais, "A solution to Plato's problem: the Latent Semantic Analysis theory of acquisition, induction and representation of knowledge," Psychological Review, vol. 104, pp. 211-240, 1997.

[24] D. M. Blei, A. Y. Ng, and M. I. Jordan, "Latent Dirichlet Allocation," Journal of Machine Learning Research, vol. 3, pp. 993-1022, 2003.

[25] T. Holmer, A. Kienle, and M. Wessner, "Explicit Referencing in Learning Chats: Needs and Acceptance," in Innovative Approaches for Learning and Knowledge Sharing, First European Conference on Technology Enhanced Learning, EC-TEL 2006, Crete, Greece, 2006, pp. $170-184$.

[26] S. Trausan-Matu and T. Rebedea, "A Polyphonic Model and System for Inter-animation Analysis in Chat Conversations with Multiple Participants," in 11th Int. Conf. Computational Linguistics and Intelligent Text Processing (CICLing 2010), Iasi, Romania, 2010, pp. 354-363.

[27] M. Galley and K. McKeown, "Improving Word Sense Disambiguation in Lexical Chaining," in 18th International Joint Conference on Artificial Intelligence (IJCAI'03), Acapulco, Mexico, 2003, pp. 14861488.

[28] G. A. Miller, "WordNet: A Lexical Database for English," Communications of the ACM, vol. 38, pp. 39-41, 1995.

[29] B. Sagot, "WordNet Libre du Francais (WOLF)," ed. Paris: INRIA, 2008

[30] T. Hastie, R. Tibshirani, and J. Friedman, The Elements of Statistical Learning. New York, NY: Springer, 2009.

[31] H. Lefebvre, Rhythmanalysis: Space, Time and Everyday Life. London, UK: Continuum, 2004.

[32] The New Harvard Dictionary of Music. Cambridge, MA: Harvard University Press, 1986.

[33] G. A. Miller, "The Magical Number Seven, Plus or Minus Two: Some Limits on our Capacity for Processing Information," Psychological Review, vol. 63, pp. 81-97, 1956.

[34] G. Upton and I. Cook, A Dictionary of Statistics. Oxford: Oxford University Press, 2008.

[35] C. E. Shannon, "A Mathematical Theory of Communication," The Bell System Technical Journal, vol. 27, pp. 379-423 \& 623-656, 1948.

[36] C. D. Manning, P. Raghavan, and H. Schütze, Introduction to Information Retrieval vol. 1. Cambridge, UK: Cambridge University Press, 2008

[37] R. M. Fano, Transmission of Information: A Statistical Theory of Communication. Cambridge, MA: MIT Press, 1961.

[38] A. J. Stent and J. F. Allen, "Annotating Argumentation Acts in Spoken Dialogue," University of Rochester. Computer Science Department, Rochester, New York, NY2000.

[39] D. Lupan, M. Dascalu, S. Trausan-Matu, and P. Dessus, "Analyzing emotional states induced by news articles with Latent Semantic Analysis," in 15th Int. Conf. on Artificial Intelligence: Methodology, Systems, Applications (AIMSA 2012), Varna, Bulgaria, 2012, pp. $59-68$. 